\title{
From 1/f Noise to Multifractal Cascades in Heartbeat Dynamics
}

\author{
Plamen Ch. Ivanov, ${ }^{1,2}$, Luís A. Nunes Amaral ${ }^{1,2}$, Ary L. Goldberger ${ }^{2}$, \\ Shlomo Havlin ${ }^{3}$, Michael G. Rosenblum ${ }^{4}$, H. Eugene Stanley ${ }^{1}$, Zbigniew R. Struzik ${ }^{5}$ \\ ${ }^{1}$ Center for Polymer Studies and Department of Physics, Boston University, Boston, MA 02215 \\ ${ }^{2}$ Harvard Medical School, Beth Israel Deaconess Medical Center, Boston, MA 02215 \\ ${ }^{3}$ Gonda Goldschmid Center and Department of Physics, Bar-Ilan University, Ramat Gan, Israel \\ ${ }^{4}$ Department of Physics, Potsdam University, D-14415 Potsdam, Germany \\ ${ }^{5}$ Centre for Mathematics and Computer Science, Kruislaan 413, NL-1098 SJ Amsterdam, The \\ Netherlands
}

\begin{abstract}
We explore the degree to which concepts developed in statistical physics can be usefully applied to physiological signals. We illustrate the problems related to physiologic signal analysis with representative examples of human heartbeat dynamics under healthy and pathologic conditions. We first review recent progress based on two analysis methods, power spectrum and detrended fluctuation analysis, used to quantify long-range power-law correlations in noisy heartbeat fluctuations. The finding of power-law correlations indicates presence of scale-invariant, fractal structures in the human heartbeat. These fractal structures are represented by self-affine cascades of beat-to-beat fluctuations revealed by wavelet decomposition at different time scales. We then describe very recent work that quantifies multifractal features in these cascades, and the discovery that the multifractal structure of healthy dynamics is lost with congestive heart failure. The analytic tools we discuss may be used on a wide range of physiologic signals.
\end{abstract}


Physiologic signals are generated by complex self-regulating systems that process inputs with a broad range of characteristics [1-3]. Many physiological time series are extremely inhomogeneous and nonstationary, fluctuating in an irregular and complex manner. An important question is whether the "heterogeneous" structure of physiologic time series arises trivially from external and intrinsic perturbations which push the system away from a homeostatic set point. An alternative hypothesis is that the fluctuations are, at least in part, due to the underlying dynamics of the system. The key problem is how to decompose subtle fluctuations (due to intrinsic physiologic control) from other nonstationary trends associated with external stimuli. Till recently, the analysis of the fractal properties of such fluctuations has been restricted to second order linear characteristics such as the power spectrum and the two-point autocorrelation function. These analyses reveal that the fractal behavior of healthy, free-running physiological systems is often characterized by $1 / f$-like scaling of the power spectra [4-8]. Monofractal signals, however, are homogeneous and have "linear" properties. Many physiologic time series - such as heartbeat interval sequences-are in fact inhomogeneous, suggesting that different parts of the signal have different scaling properties. In addition, there is evidence that heartbeat dynamics exhibits nonlinear properties [9-15]. Such features are often associated with multifractal behavior. Up to now, robust demonstration of multifractality for nonstationary time series has been hampered by problems related to a drastic bias in the estimate of the singularity spectrum due to diverging negative moments. Moreover, the classical approaches based on the box-counting technique and structure function formalism fail when a fractal function is composed of a multifractal singular part embedded in regular polynomial behavior [16]. By means of a waveletbased multifractal formalism, we show that healthy human heartbeat dynamics exhibits even higher complexity (than previously expected from the finding of fractal $1 /$ f scaling) which is characterized by a broad multifractal spectrum [17]. 


\section{INTRODUCTION}

In recent years the study of the statistical properties of heartbeat interval sequences has attracted the attention of researchers from different fields [18-22]. Analysis has focused extensively on interbeat interval variability as an important quantity to help elucidate possibly

nonhomeostatic physiologic variability because: (i) the heart rate is under direct neuroautonomic control, (ii) interbeat interval variability is readily measured by noninvasive means, and (iii) analysis of these heart rate dynamics may provide important practical diagnostic and prognostic information. Figure 1 shows a cardiac interbeat time series - the output of a spatially and temporally integrated neuroautonomic control system. The time series shows "erratic" fluctuations and "patchiness". These fluctuations are usually ignored in conventional studies which focus on averaged quantities. In fact, these fluctuations are often labeled as "noise" to distinguish them from the true "signal" of interest. Generally, in the conventional approach it is assumed that there is no meaningful structure in apparent noise and, therefore, one does not expect to gain any understanding about the underlying system through the study of these fluctuations. However, by adapting and extending methods developed in modern statistical physics and nonlinear dynamics, we find that the physiologic fluctuations shown in Fig. 1 exhibit an unexpected hidden scaling structure [6,13,17,23-25]. Furthermore, the dynamical patterns of these fluctuations and the associated scaling features change with pathological perturbations. These findings raise the possibility that understanding the origin of such temporal structures and their alterations with disease $(a)$ may elucidate certain basic aspects of heart rate control mechanisms, and (b) may have potential for clinical monitoring.

\section{1/F FLUCTUATIONS IN HEARTBEAT DYNAMICS}

A quantity widely used to measure correlations in a time series is the power spectrum, which measures the relative frequency content of a signal. Fourier and related power spec- 
trum analysis have proved particularly useful for recognizing the existence and role of characteristic frequencies (time scales) in cardiac dynamics. The analysis of heart beat fluctuations focused initially on short time oscillations associated with breathing and blood pressure as well as other control [20,21]. Studies of longer heartbeat records revealed $1 / \mathrm{f}$-like scale-free behavior $[4,5]$. A power spectrum calculation assumes that the signal studied is stationary $[26,27]$, and when applied to nonstationary time series can lead to misleading results. However, time series of beat-to-beat $(\mathrm{RR})$ heart rate intervals obtained from digitized electrocardiograms are typically nonstationary and fluctuate in an irregular manner in healthy subjects, even at rest [Fig. 1b] [28,29]. Because of this property, researchers were faced with the task to consider only portions of the data and to test these portions for stationarity before performing power spectrum analysis.

To illustrate the limitations of the power spectrum analysis for nonstationary time series, we consider 6 hour records ( $n \approx 10^{4}$ beats) of interbeat intervals for a healthy subject during sleep and wake activity. We show that there is no true $1 / f$ power spectrum for the interbeat intervals in the real heart. Instead, we find that the power spectrum of the interbeat intervals has different regimes with different scaling behavior and that the rounded crossover between the different regimes is the reason why it seems, to first approximation, to scale as $1 / f$ [Fig. 2].

Recent analyses of very long time series (up to $24 \mathrm{~h}: n \approx 10^{5}$ beats) show that under healthy conditions, interbeat interval increments $I(n)$ exhibit power-law anticorrelations [6]. Since $I(n)$ is stationary, we can apply standard spectral analysis techniques [Fig. 3] and we show that true scaling does exist.

The fact that the log-log plot of the power spectrum $S_{I}(f)$ vs. $f$ is linear implies:

$$
S_{I}(f) \sim f^{-\beta}
$$

The exponent $\beta$ is related to the mean fluctuation function exponent $\alpha$ by $\beta=2 \alpha-1$ $[30,31]$ and can serve as an indicator of the presence and type of correlations: (i) If $\beta=0$, there is no correlation in the time series $I(n)$ ("white noise"); (ii) If $0<\beta<1$, then $I(n)$ 
is correlated such that positive values of $I$ are likely to be close (in time) to each other, and the same is true for negative $I$ values; (iii) If $-1<\beta<0$, then $I(n)$ is also correlated. However, the values of $I$ are organized such that positive and negative values are more likely to alternate in time ("anticorrelation") [30].

For interbeat interval increments from records of healthy subjects we obtain $\beta \simeq-1$, suggesting non-trivial power-law long-range correlations in the heartbeat. Furthermore, the anti-correlation properties of $I$ indicated by the negative $\beta$ are consistent with a nonlinear feedback system that "kicks" the heart rate away from extremes [32,33]. This tendency, however, does not only operate locally on a beat-to-beat basis, but over a wide range of time scales up to thousands of beats [Fig. 3]. The emergence of such scale-invariant properties in the seemingly "noisy" heart beat fluctuations is believed to be a result of highly complex, nonlinear mechanisms of physiologic control $[31,36]$.

Extracting increments from a time series is only a first step in effectively treating problems related to nonstationarities. Note that the power spectrum of the increments in the heartbeat intervals [Fig. 3] does not distinguish between wake and sleep dynamics. One needs to do better - e.g. by taking into account the presence of polynomial trends in the times series. We discuss such an approach in the following Section.

\section{MONOFRACTAL ANALYSIS: LONG-RANGE ANTICORRELATIONS IN THE HEARTBEAT FLUCTUATIONS}

Recently the detrended fluctuation analysis (DFA) method [37] was introduced to detect long-range correlations in physiological fluctuations when these are embedded in a seemingly nonstationary time series. The advantage of the DFA method over conventional methods, such as power spectrum analysis, is that it avoids the spurious detection of apparent longrange correlations that are an artifact of nonstationarity related to linear and higher order polynomial trends in the data. The essence of the DFA method is as follows: the average root-mean-square fluctuation function $F(n)$ is obtained after integrating and detrending the 
data, i.e., subtracting the local polynomial trend in a box of size $n$ data points. The powerlaw relation between $F(n)$ and the number of data points $n$ in a box indicates the presence of scaling: the fluctuations can be characterized by a scaling exponent $\alpha$, a self-similarity parameter, defined as $F(n) \sim n^{\alpha}$. The DFA method has been tested on control time series of "built-in" long-range correlations with superposition of a nonstationary external trend [38]. It has also been successfully applied to detect long-range correlations in human gait, ion channel kinetics, and highly heterogeneous DNA sequences [7,8,37,39-41]. Of note is a recent independent review of fractal fluctuation analysis methods which determined that DFA was one of the most robust methods [42].

It is known that circadian rhythms are associated with periodic changes in key physiological processes $[3,36,44]$. Typically the differences in the cardiac dynamics during sleep and wake phase are reflected in the average and standard deviation of the interbeat intervals $[43,44]$. Such differences can be systematically observed from plots of the interbeat intervals recorded from subjects during sleep and wake [Fig. 1]. In recent studies we have reported on sleep-wake differences in the distributions of the amplitudes of the fluctuations in the interbeat intervals — a surprising finding indicating higher probability for larger amplitudes during sleep $[13,24,45]$. Next, we ask the question if there are characteristic differences in the scaling behavior between sleep and wake cardiac dynamics. We hypothesize that sleep and wake changes in cardiac control may occur on all time scales and thus could lead to systematic changes in the scaling properties of the heartbeat dynamics. Elucidating the nature of these sleep-wake rhythms could lead to a better understanding of the neuroautonomic mechanisms of cardiac regulation.

To answer this question we apply the detrended fluctuation analysis (DFA) method. We analyze 30 datasets — each with $24 \mathrm{~h}$ of interbeat intervals — from 18 healthy subjects and 12 patients with congestive heart failure [46]. We analyze the nocturnal and diurnal fractions of the dataset of each subject, which correspond to the $6 \mathrm{~h}(n \approx 22,000$ beats) from midnight to $6 \mathrm{am}$ and noon to $6 \mathrm{pm}$. These periods incorporate the segments with lowest and highest heart rate in the time series, which we and others found to be the best indirect 
marker of sleep $[43,44]$. We find that at scales above $\approx 1 \min (n>60)$ the data during wake hours display long-range correlations over two decades with average exponents $\alpha_{W} \approx 1.05$ for the healthy group and $\alpha_{W} \approx 1.2$ for the heart failure patients. For the sleep data we find a systematic crossover at scale $n \approx 60$ beats followed by a scaling regime extending over two decades characterized by a smaller exponent: $\alpha_{S} \approx 0.85$ for the healthy and $\alpha_{S} \approx 0.95$ for the heart failure group [Fig. 4a,c]. Although the values of the sleep and wake exponents vary from subject to subject, we find that for all individuals studied, the heartbeat dynamics during sleep are characterized by a smaller exponent [47].

This analysis suggests that the observed sleep-wake scaling differences are due to intrinsic changes in the cardiac control mechanisms for the following reasons: (i) The DFA method removes the "trends" in the interbeat interval signal which are due, at least in part, to activity, and quantifies the fluctuations along the trends. (ii) Responses to external stimuli should give rise to a different type of fluctuations having characteristic time scales, i.e. frequencies related to the stimuli. However, fluctuations in both diurnal and nocturnal cardiac dynamics exhibit scale-free behavior. (iii) The weaker anticorrelated behavior observed for all wake phase records cannot be simply explained as a superposition of stronger anticorrelated sleep dynamics and random noise of day activity. Such noise would dominate at large scales and should lead to a crossover with an exponent of 1.5. However, such crossover behavior is not observed in any of the wake phase datasets [Fig. 4]. Rather, the wake dynamics are typically characterized by a stable scaling regime up to $n=5 \times 10^{3}$ beats.

To test the robustness of our results, we analyze 17 datasets from 6 cosmonauts during long-term orbital flight on the Mir space station under the extreme conditions of zero gravity and high stress activity [48]. Each dataset contains continuous periods of $6 \mathrm{~h}$ data under both sleep and wake conditions. We find that for all cosmonauts the heartbeat interval series exhibit long-range correlations with scaling exponents consistent with those found for the healthy terrestrial group: $\alpha_{W} \approx 1.04$ for the wake phase and $\alpha_{S} \approx 0.82$ for the sleep phase. The values of these exponents indicate that the fluctuations in the interbeat intervals are anticorrelated for the wake phases and even stronger anticorrelated for the sleep phase. This 
sleep-wake scaling difference is observed not only for the group averaged exponents but for each individual cosmonaut dataset [Fig. 4b]. Moreover, the scaling differences are persistent in time, since records of the same cosmonaut taken on different days (ranging from the 3rd to the 158th day in orbit), exhibit a higher degree of anticorrelation in sleep.

Thus, the larger values for the wake phase scaling exponents observed for healthy subjects cannot be a trivial artifact of activity. Furthermore, the larger value of the average wake exponent for the heart failure group compared to the other two groups cannot be attributed to external stimuli either, since patients with severe cardiac disease are strongly restricted in their physical activity. We note, however, that the average sleep-wake scaling difference remains the same $(\approx 0.2)$ for all three groups. Such sleep-wake changes in the scaling characteristics may indicate different regimes of intrinsic neuroautonomic regulation of the cardiac dynamics, which may "switch" on and off in accordance with circadian rhythms. A very recent study confirms our finding of lower value for the scaling exponent during sleep and shows that different stages of sleep (e.g. light sleep, deep sleep, rapid eye movement stages) could be associated with different correlations in the heartbeat fluctuations [49]. The findings of stronger anticorrelations [47], as well as higher probability for larger heartbeat fluctuations during sleep $[13,24,45]$ are of interest from a physiological viewpoint, since they suggest that the observed dynamical characteristics in the heartbeat fluctuations during sleep and wake phases are related to intrinsic mechanisms of neuroautonomic control, and support a reassessment of the sleep as a surprisingly active dynamical state. The finding of scaling features in the human heartbeat and thier change with disease or sleep-wake transition have motivated new modeling approaches which may lead to better understanding the underlying control mechanisms of heartrate regulation [33].

Before concluding this Section we note that recent work [34] provides evidence of surprising complexity present in the temporal organization of the heterogeneities (e.g. trends) in human heartbeat dynamics. Trends in the interbeat interval signal are traditionally associated with external stimuli. To probe the temporal organization of such heterogeneities we introduce a segmentation algorithm [35] and find that the lengths of segments with different 
local mean heart rates follow a power-law distribution. This scale-invariant structure is not a simple consequence of the long-range correlations present in the heartbeat fluctuations discussed in this Section. These new findings suggest that relevant physiological information may be hidden in the heterogeneities of the heartbeat time series, the understanding of which remains an open question.

\section{SELF-SIMILAR CASCADES IN THE HEARTBEAT FLUCTUATIONS}

Many simple systems in nature have correlation functions that decay with time in an exponential way. For systems comprised of many interacting subsystems, physicists discovered that such exponential decays typically do not occur. Rather, correlation functions were found to decay with a power-law form. The implication of this discovery is that in complex systems, there is no single characteristic time [50-52]. If correlations decay with a power-law form, we say the system is "scale-free" because there is no characteristic scale associated with a power law. Since at large time scales a power law is always larger than an exponential function, correlations described by power laws are termed "long-range" correlations - they are of longer range than exponentially-decaying correlations.

The findings of long-range power-law correlations [23,47] and the recently reported scaling in the distributions of heartbeat fluctuations $[13,45]$ (i.e. "data collapse" of the distributions for different time scales) suggest the absence of a characteristic scale and indicate that the underlying dynamical mechanisms regulating the healthy heartbeat have statistical properties which are similar on different time scales. Such statistical self-similarity is an important characteristic of fractal objects [53]. However, how can this purported fractal structure be "visualized" in the seemingly erratic and noisy heartbeat fluctuations? The wavelet decomposition of beat-to-beat heart rate signals can be used to provide a visual representation of this fractal structure [Fig. 5]. The brighter colors indicate larger values of the wavelet amplitudes (corresponding to large heartbeat fluctuations) and white tracks represent the wavelet transform maxima lines. The structure of these maxima lines shows the evolution 
of the heartbeat fluctuations with scale and time. The wavelet analysis performed with the second derivative of the Gaussian (the Mexican hat) as an analyzing wavelet uncovers a hierarchical scale-invariance [Fig. 5 (top panel)], which is characterized by the stability of the scaling form observed for the distributions and the power-law correlations [13,23,47]. The plots reveal a self-affine cascade formed by the maxima lines - a magnification of the central portion of the top panel shows similar branching patterns [Fig. 5 (lower panel)]. Such fractal cascade results from the interaction of many nonlinearly coupled physiological components, operating on different scales (polynomial trends due to daily activity are filtered out).

Thus the wavelet transform, with its ability to remove local trends and to extract interbeat variations on different time scales, enables us to identify fractal patterns (arches) in the heartbeat fluctuations even when the signals change as a result of background interference. Analysis of data from pathologic conditions (e.g. sleep apnea) show a breakdown these patterns [24]. Fractal characteristics of cardiac dynamics and other biological signals can be usefully studied with the generalized multifractal formalism based on the wavelet transform modulus maxima method which we discuss in the next Section.

\section{MULTIFRACTALITY: NONSTATIONARITY IN LOCAL SCALING}

Monofractal signals are homogeneous in the sense that they have the same scaling properties, characterized locally by a single singularity exponent $h_{0}$, throughout the entire signal [52-57]. Therefore monofractal signals can be indexed by a single global exponent-the Hurst exponent $H \equiv h_{0}[58]$ — which suggests that they are stationary from viewpoint of their local scaling properties. On the other hand, multifractal signals, can be decomposed into many subsets — possibly infinitely many — characterized by different local Hurst exponents $h$, which quantify the local singular behavior and thus relate to the local scaling of the time series [Fig. 6]. Thus multifractal signals require many exponents to fully characterize their scaling properties $[53,55,57]$ and are intrinsically more complex, and inhomogeneous, than monofractals. 
The statistical properties of the different subsets characterized by these different exponents $h$ can be quantified by the function $D(h)$, where $D\left(h_{o}\right)$ is the fractal dimension of the subset of the time series characterized by the local Hurst exponent $h_{o}[53,55,57,59-61]$. Thus, the multifractal approach for signals, a concept introduced in the context of multiaffine functions $[62,63]$, has the potential to describe a wide class of signals that are more complex then those characterized by a single fractal dimension (such as classical 1/f noise).

In a recent study, we establish the relevance of the multifractal formalism for the description of a physiological signal — the human heartbeat [17]. The motivation for our work is not merely looking for yet another example of multifractality, this time in the biological sciences. In fact, if we consider the neuroautonomic control mechanisms responsible for the generation of heartbeats, it is natural to expect the need for multifractal concepts for their description, since the heartbeats are a result of the interaction of many physiological components operating on different time scales. These interactions are nonlinear and self-regulating (through feedback control), leading to the nonlinear character of the output signal and to the heterogeneous features of heartbeat time series.

In contrast, the assumption of heartbeat monofractality — which has been the scope of studies in the field so far - is unrealistic because the monofractal hypothesis assumes that the scaling properties of the signal are the same throughout time, and are characterized by the same local Hurst exponent $h$ [Fig. 7c]. However, inspection of heartbeat signals shows them to be heterogeneous and suggests they might require more exponents for their description. Since the power spectrum and the correlation analysis (DFA method) can measure only one exponent characterizing a given signal, these methods are more appropriate for the study of monofractal signals. Moreover, the power spectrum and the correlation analysis reflect only the linear characteristics, while the heartbeat dynamics exhibits nonlinear properties. Thus the multifractal analysis may reveal new information on the nature of the nonlinearity encoded in the Fourier phases [Fig. 12].

The first problem, therefore, is to extract the local value of $h$. To this end we use methods derived from wavelet theory [64]. The properties of the wavelet transform make 
wavelet methods attractive for the analysis of complex nonstationary time series such as one encounters in physiology [13]. In particular, wavelets can remove polynomial trends that could lead box-counting techniques to fail to quantify the local scaling of the signal [65]. Additionally, the time-frequency localization properties of the wavelets makes them particularly useful for the task of revealing the underlying hierarchy in the cascade of fluctuations [Fig. 5] that governs the temporal distribution of the local Hurst exponents. Hence, the wavelet transform enables a reliable multifractal analysis [65]. As the analyzing wavelet, we use derivatives of the Gaussian function, which allows us to estimate the singular behavior and the corresponding exponent $h$ at a given location in the time series. The higher the order $n$ of the derivative, the higher the order of the polynomial trends removed and the better the detection of the temporal structure of the local scaling exponents in the signal.

The concept of multifractality is exemplified in Fig. 7a,b for a heartbeat intervals record from a healthy subject. The heterogeneity of the healthy heartbeat is represented by the broad range of local Hurst exponents $h$ (colors) present and the complex temporal organization of the different exponents. The middle and bottom panels illustrate the different fractal structure of two subsets of the time series characterized by different local Hurst exponents. The value of the local Hurst exponent for each subset is represented with a shade of green and red, respectively. The two subsets display different temporal structures which can be quantified by different fractal dimension $D(h)$. The healthy signal is represented by a multicolor plot, reflecting multifractal behavior through the variety of values for the local Hurst exponents. In contrast, fractional Brownian motion (a monofractal signal) is essentially monochromatic indicating that the local Hurst exponent $h$ is the same throughout the signal [Fig. 7c].

\section{MULTIFRACTALITY IN HEARTBEAT DYNAMICS}

We evaluate the local exponent $h$ through the modulus of the maxima values of the wavelet transform at each point in the time series using the wavelet transform modulus max- 
ima method [65]. However, heartbeat time series contain densely packed, non-isolated singularities which unavoidably affect each other in the time-frequency decomposition. Therefore, rather than evaluating the distribution of the inherently unstable local singularity exponents (as shown in color in Fig. 7), we estimate the scaling of an appropriately chosen global measure - a partition function $Z_{q}(a)$, which is defined as the sum of the $q^{\text {th }}$ powers of the local maxima of the modulus of the wavelet transform coefficients at scale $a$. For each scale $a$ these local maxima values are traced along the maxima lines obtained after the wavelet decomposition of the heartbeat signal (maxima lines appear in bright/white color in Fig. 5). As analyzing wavelet we use the 3rd derivative of the Gaussian function. For small scales, we expect

$$
Z_{q}(a) \sim a^{\tau(q)} .
$$

For certain values of $q$, the exponents $\tau(q)$ have familiar meanings. In particular, $\tau(2)$ is related to the scaling exponent of the Fourier power spectra, $S(f) \sim 1 / f^{\beta}$, as $\beta=$ $2+\tau(2)$. For positive $q, Z_{q}(a)$ reflects the scaling of the large fluctuations and strong singularities, while for negative $q, Z_{q}(a)$ reflects the scaling of the small fluctuations and weak singularities $[55,57]$. Thus, the scaling exponents $\tau(q)$ can reveal different aspects of cardiac dynamics [Fig. 8] . Monofractal signals display a linear $\tau(q)$ spectrum, $\tau(q)=q H-1$, where $H$ is the global Hurst exponent. For multifractal signals, $\tau(q)$ is a nonlinear function: $\tau(q)=q h(q)-1$, where $h(q) \equiv d \tau(q) / d q$ is not constant.

A previous obstacle to the determination of the multifractal spectrum of a time series has been the calculation of the negative moments. Until the application of the wavelet modulus maxima method, it was not possible to estimate $Z_{q}(a)$ for $q<0$. We calculate $\tau(q)$ for moments $q=-5,4, \ldots, 0, \ldots, 5$ and scales $a=2 \times 1.15^{i}, i=0, \ldots, 41$ from 6 hours records obtained from a healthy subject and a subject with congestive heart failure. In Fig. 8a,b we display the calculated values of $Z_{q}(a)$ for scales $a>8$. The top curve corresponds to $q=-5$, the middle curve (shown heavy) to $q=0$ and the bottom curve to $q=5$. The exponents $\tau(q)$ are obtained from the slope of the $Z_{q}(a)$ curves in the region 
$16<a<700$, thus eliminating the influence of any residual small scale random noise due to electrocardiogram signal pre-processing as well as extreme, large scale fluctuations of the signal. A monofractal signal would correspond to a straight line for $\tau(q)$, while for a multifractal signal $\tau(q)$ is nonlinear. Note the clear differences between the $\tau(q)$ curves for healthy and heart failure records [Fig. 8c]. The constantly changing curvature of the $\tau(q)$ curves for the healthy records, suggests multifractality. In contrast, $\tau(q)$ is almost linear for the congestive heart failure subject, indicating monofractality.

We analyze both daytime (12:00 to 18:00) and nighttime (0:00 to 6:00) heartbeat time series records of healthy subjects, and the daytime records of patients with congestive heart failure. These data were obtained by Holter monitoring. Our database includes 18 healthy subjects (13 female and 5 male, with ages between 20 and 50, average 34.3 years), and 12 congestive heart failure subjects ( 3 female and 9 male, with ages between 22 and 71, average 60.8 years) in sinus rhythm $[46]$.

Next, we obtain the fractal dimension $D(h)$. It is related to $\tau(q)$ through a Legendre transform,

$$
D(h)=q \frac{d \tau(q)}{d q}-\tau(q)
$$

For all healthy subjects, we find that $\tau(q)$ is a nonlinear function [Fig. 8c and Fig. 9a], which indicates that the heart rate of healthy humans is a multifractal signal. Figure $9 \mathrm{~b}$ shows that for healthy subjects, $D(h)$ has nonzero values for a broad range of local Hurst exponents $h$. The multifractality of healthy heartbeat dynamics cannot be explained by activity, as we analyze data from subjects during nocturnal hours. Furthermore, this multifractal behavior cannot be attributed to sleep-stage transitions, as we find multifractal features during daytime hours as well [66]. The range of scaling exponents $-0<h<0.3-$ with nonzero fractal dimension $D(h)$, suggests that the fluctuations in the healthy heartbeat dynamics exhibit anti-correlated behavior $(h=1 / 2$ corresponds to uncorrelated behavior while $h>1 / 2$ corresponds to correlated behavior).

In contrast, we find that heart rate data from subjects with a pathological condition - 
congestive heart failure - show a clear loss of multifractality [Figs. 9a,b]. For the heart failure subjects, $\tau(q)$ is close to linear and $D(h)$ is non-zero only over a very narrow range of exponents $h$ indicating monofractal behaviour [Fig. 9].

Our results show that, for healthy subjects, local Hurst exponents in the range $0.07<$ $h<0.17$ are associated with fractal dimensions close to one. This means that the subsets characterized by these local exponents are statistically dominant. On the other hand, for the heart failure subjects, we find that the statistically dominant exponents are confined to a narrow range of local Hurst exponents centered at $h \approx 0.22$. These results suggest that for heart failure the fluctuations are less anti-correlated than for healthy dynamics since the dominant scaling exponents $h$ are closer to $1 / 2$. Thus, our findings support previous reports of long-range anti-correlations in healthy heartbeat fluctuations [see caption to Fig. 9] [23].

We present color panels with the local Hurst $h$ exponent for 6 healthy individuals [Fig. 10] and 6 subjects with congestive heart failure [Fig. 11]. Each panel represents $6 \mathrm{~h}$ long record. The color code for these panels is the following: with increasing value of $h$, the spectrum goes from red to green to blue. A wider range of colors indicates a higher degree of multifractality. For this reason, records from healthy individuals should be more polychromatic. On the other hand, records from heart failure patients should be more monochromatic (with a single color predominating), indicating loss of multifractality. In addition, the color spectrum for the healthy individuals is shifted to the red and for the heart failure patients is shifted to the blue. This is in agreement with the results in Fig. 9 where the peak of the multifractal spectrum $D(h)$ is centered at smaller values of $h$ for the healthy group and at larger values of $h$ for the heart failure group. These findings may have a potential for diagnosis [67].

\section{MULTIFRACTALITY AND NONLINEARITY}

The multifractality of heart beat time series also enables us to quantify the greater complexity of the healthy dynamics compared to pathological conditions. Power spectrum and detrended fluctuation analysis define the complexity of heart beat dynamics through its 
scale-free behavior, identifying a single scaling exponent as an index of healthy or pathologic behavior. Hence, the power spectrum is not able to quantify the greater level of complexity of the healthy dynamics, reflected in the heterogeneity of the signal. On the other hand, the multifractal analysis reveals this new level of complexity by the broad range of exponents necessary to characterize the healthy dynamics [Fig. 9]. Moreover, the change in shape of the $D(h)$ curve for the heart failure group may provide insights into the alteration of the cardiac control mechanisms due to this pathology.

To further study the complexity of the healthy dynamics, we perform two tests with surrogate time series. First, we generate a surrogate time series by shuffling the interbeat interval increments of a record from a healthy subject. The new signal preserves the distribution of interbeat interval increments but destroys the long-range correlations among them. Hence, the signal is a simple random walk, which is characterized by a single Hurst exponent $H=1 / 2$ and exhibits monofractal behavior [Fig. 12a]. Second, we generate a surrogate time series by performing a Fourier transform on a record from a healthy subject, preserving the amplitudes of the Fourier transform but randomizing the phases, and then performing an inverse Fourier transform. This procedure eliminates nonlinearities, preserving only the linear features of the original time series. The new surrogate signal has the same $1 / f$ behavior in the power spectrum as the original heart beat time series; however it exhibits monofractal behavior [Fig. 12a]. We repeat this test on a record of a heart failure subject. In this case, we find a smaller change in the multifractal spectrum [Fig. 12b]. The results suggest that the healthy heartbeat time series contains important phase correlations canceled in the surrogate signal by the randomization of the Fourier phases, and that these correlations are weaker in heart failure subjects. Furthermore, the tests indicate that the observed multifractality is related to nonlinear features of the healthy heartbeat dynamics. A number of recent studies have tested for nonlinear and deterministic properties in recordings of interbeat intervals $[9-11,14,15]$. Our results suggest an explicit relation between the nonlinear features (represented by the Fourier phase interactions) and the multifractality of healthy cardiac dynamics [Fig. 12]. 


\section{SUMMARY AND OPEN QUESTIONS}

The discovery of multifractality in a physiological time series and its breakdown with pathology is significant from a number of perspectives.

First, contemporary analysis of heartbeat fluctuations, and the study of physiological time series in general, have emphasized two important, but apparently unconnected properties: (i) the presence of nonlinearities and (ii) 1/f-behavior (monofractality). The monofractal hypothesis assumes that the scaling properties of the signal are the same throughout. Yet the heterogeneous nature of the heartbeat interval time series clearly indicates nonlinear features. The finding of a multifractal mechanism for heartrate control provides a unifying connection between nonlinear and fractal properties and, indeed indicates that they are aspects of a more fundamental type of mechanism. In particular, we show that both the multifractal character and the nonlinear properties of the signal are encoded in the Fourier phases [Fig. 12]. The origin and nature of these Fourier phase interactions is an open question.

Second, our analysis indicates that the healthy heartbeat is described by a broad range of scaling exponents $h$ with a well-defined set of bounding parameters, $h_{\min }$ and $h_{\max }$. Furthermore, certain exponents appear to be "forbidden" $\left(h<h_{\min }\right.$ and $\left.h>h_{\max }\right)$ and the exponents present occur with a given structure characterized by the function $D(h)$.

Third, our findings may lead to new diagnostic applications. Further detailed studies on a larger number of datasets are needed to establish the advantages of given methods compared to others and to find optimal combinations of methods for diagnostic and prognostic purposes.

Fourth, our analysis is based on a "microscopic" approach which can identify the statis-

tical properties of the self-affine cascade of heartbeat fluctuations at different scales [Fig. 5]. Our finding of multifractality quantifies the complex dynamics of this cascade and suggests that a multiplicative mechanism might be the origin of this phenomena. The detailed features of the cascades and how they relate to other processes with cascades (e.g., turbulence) 
remain to be addressed.

On a more general level, our approach provides a way of testing a broad range of $1 / \mathrm{f}$-type signals to see if they represent multifractal or monofractal processes. As such, these findings should be of interest to a very wide audience given the historic interest in elucidating the nature of different types of $1 / \mathrm{f}$ noise.

Finally, from a physiological perspective, the detection of robust multifractal scaling in the heart rate dynamics is of interest because our findings raise the intriguing possibility that the control mechanisms regulating the heartbeat interact as part of a coupled cascade of feedback loops in a system operating far from equilibrium - an extraordinarily complex behavior which in physical systems has been connected with turbulence and related multiscale phenomena [68-70]. Furthermore, the present results indicate that the healthy heartbeat is even more complex than previously suspected, posing a challenge to ongoing efforts to develop realistic models of the control of heart rate and other processes under neuroautonomic regulation $[18,33,71-73]$.

\section{ACKNOWLEDGMENTS}

We thank A. Arneodo, Y. Ashkenazy, P. Bernaola-Galván, A. Bunde, P. Carpena, F. Family, U. Frisch, J.M. Hausdorff, I. Grosse, H. Herzel, V. Horváth, H. Kallabis, J.W. Kantelhardt, J. Kurths, Y. Lee, T. Lopez-Ciudad, H. Makse, C.-K. Peng, B. Rosenow, V. Schulte-Frohlinde, K.R. Sreenivasan, V. Vicsek and B.J. West for valuable discussions. This

work was supported by NIH/National Center for Research Resources (P41 RR13622), NSF, The Centers for Disease Control and Prevention, The Fetzer Institute, and The G. Harold and Leila Y. Mathers Charitable Foundation. 


\section{REFERENCES}

[1] M. F. Shlesinger, "Fractal time and $1 / f$ noise in complex systems", Ann. NY Acad. Sci. 504, 214 (1987).

[2] J.B. Bassingthwaighte, L.S. Liebovitch, and B.J. West, Fractal Physiology (Oxford Univ. Press, New York, 1994).

[3] M. Malik and A.J. Camm, eds., Heart Rate Variability (Futura, Armonk NY, 1995).

[4] M. Kobayashi and T. Musha, " $1 / f$ fluctuation of heartbeat period", IEEE Trans. Biomed. Eng. 29, 456 (1982).

[5] J.P. Saul, P. Albrecht, D. Berger, and R.J. Cohen, Computers in Cardiology (IEEE Computer Society Press, Washington DC), 419 (1987).

[6] C.-K. Peng, J. Mietus, J.M. Hausdorff, S. Havlin, H. Eugene Stanley, and A.L. Goldberger, "Long-range Anti-Correlations and Gon-Gaussian Behavior of the Heartbeat", Phys. Rev. Lett. 70, 1343 (1993).

[7] J.M. Hausdorff, P.L. Purdon, C.-K. Peng, Z. Ladin, J. Y. Wei, and A.L. Goldberger, "Fractal Dynamics of Human Gait: Stability of Long-range Correlations in Stride Interval Fluctuations", J. Appl. Physiol. 80, 1448 (1996).

[8] L.S. Liebovitch, "Fractal Analysis of Channel Mechanisms", Adv. Chem. Ser. 235, 357 (1994); S.B. Lowen, L.S. Liebovitch, J.A. White, "Fractal Ion-Channel Behavior Generates Fractal Firing Patterns in Neuronal Models", Phys. Rev. E 59, 5970 (1999).

[9] J. Lefebvre, D.A. Goodings, M.V. Kamath, and E.L. Fallen, "Predictability of Normal Heart Rhythms and Deterministic Chaos", Chaos 3, 267 (1993).

[10] Y. Yamamoto, R.L. Hughson, J.R. Sutton, C.S. Houston, A. Cymerman, E.L. Fallen, and M.V. Kamath, "Operation Everest II: an Indication of Deterministic Chaos in Human Heart Rate Variability at Simulated Extreme Altitude", Biol. Cybern. 69, 205 
(1993).

[11] J.K. Kanters, N.H. Holstein-Rathlou and E. Agner, "Lack of Evidence for Lowdimensional Chaos in Heart Rate Variability", J. Cardiovasc. Electrophysiol. 5, 128 (1994).

[12] J. Kurths, A. Voss, P. Saparin, A. Witt, H.J. Kleiner, and N. Wessel, "Quantitative Analysis of Heart Rate Variability", Chaos 5, 88 (1995).

[13] P. Ch. Ivanov, M. G. Rosenblum, C.-K. Peng, J. Mietus, S. Havlin, H. E. Stanley and A. L. Goldberger, "Scaling Behaviour of Heartbeat Intervals Obtained by Wavelet-based Time-series Analysis," Nature 383, 323 (1996).

[14] G. Sugihara, W. Allan, D. Sobel and K. D. Allan, "Nonlinear Control of Heart Rate Variability in Human Infants", Proc. Natl. Acad. Sci. USA 93, 2608 (1996).

[15] C-S. Poon, and C.K. Merrill, "Decrease of Cardiac Chaos in Congestive Heart Failure", Nature 389, 492 (1997).

[16] J. F. Muzy, E. Bacry, and A. Arneodo, "Multifractal Formalism for Fractal Signals: The Structure-Function Approach versus the Wavelet-Transform Modulus-Maxima Method", Phys. Rev. E 47, 875 (1993).

[17] P. Ch. Ivanov, L. A. N. Amaral, A. L. Goldberger, S. Havlin, M. G. Rosenblum, Z. Struzik, and H. E. Stanley, "Multifractality in Human Heartbeat Dynamics", Nature 399461 (1999).

[18] M. Mackey and L. Glass, "Oscillation and Chaos in Physiological Control Systems", Science 197, 287 (1977).

[19] M.M. Wolf, G.A. Varigos, D. Hunt, and J.G. Sloman, "Sinus Arrhythmia in Acute Myocardial Infarction", Med. J. Australia 2, 52 (1978).

[20] R.I. Kitney and O. Rompelman, The Study of Heart-Rate Variability (Oxford Univ. 
Press, London, 1980).

[21] S. Akselrod, D. Gordon, F. A. Ubel, D. C. Shannon, A. C. Barger, and R. J. Cohen, "Power Spectrum Analysis of Heart Rate Fluctuation: a Quantitative Probe of Beatto-Beat Cardiovascular Control," Science 213, 220 (1981).

[22] L. Glass, P. Hunter, and A. McCulloch, eds., Theory of Heart (Springer Verlag, New York, 1991).

[23] C.-K. Peng, S. Havlin, H. E. Stanley, and A. L. Goldberger, "Quantification of scaling exponents and crossover phenomena in nonstationary heartbeat time series," in Proc. NATO dynamical disease conference, edited by Glass L. Chaos 5, 82 (1995).

[24] P.Ch. Ivanov, M.G. Rosenblum, C.-K. Peng, J. Mietus, S. Havlin, H.E. Stanley, and A.L. Goldberger, "Scaling and Universality in Heart Rate Variability Distributions," Physica A 249, 587 (1998).

[25] Y. Ashkenazy, P.Ch. Ivanov, S. Havlin, C.-K. Peng, A.L. Goldberger, and H.E. Stanley, "Magnitude and Sign Correlations in Heartbeat Fluctuations," Phys. Rev. Lett. 86, $1900(2001)$.

[26] D. Panter, Modulation, noise and spectral analysis (McGraw-Hill, New York, 1965).

[27] R. L. Stratonovich, Topics in the theory of random noise, vol. I (Gordon and Breach, New York, 1981).

[28] R.I. Kitney, D. Linkens, A.C. Selman, and A.A. McDonald, Automedica 4, 141 (1982).

[29] A.L. Goldberger, "Non-linear Dynamics for Clinicians: Chaos Theory, Fractals, and Complexity at the Bedside", Lancet 347, 1312 (1996).

[30] S. Havlin et al.. "Random Multiplicative Processes and Transport in Structures with Correlated Spatial Disorder", Phys. Rev. Lett. 61, 1438 (1988).

[31] M.F. Shlesinger and B.J. West, Random Fluctuations and Pattern Growth: Experiments 
and Models (Kluwer Academic Publishers, Boston, 1988).

[32] M. N. Levy, "Sympathetic-Parasympathetic Interactions in the Heart", Circ. Res. 29, 437 (1971).

[33] P. Ch. Ivanov, L.A.N. Amaral, A.L. Goldberger, and H.E. Stanley, "Stochastic Feedback and the Regulation of Biological Rhythms", Europhys. Lett. 43, 363 (1998).

[34] P. Bernaola-Galvan, P.Ch. Ivanov, L.A.N. Amaral, and H.E. Stanley, "ScaleInvariance in the Nonstationarity of Physiological Signals", (http://xxx.lanl.gov/condmat/0005284)

[35] P. Bernaola-Galvan, I. Grosse, P. Carpena, J.L. Oliver, R. Roman-Roldan, H.E. Stanley, "Finding Borders between Coding and Noncoding DNA Regions by an Entropic Segmentation Method", Phys. Rev. Lett. 85, 1342 (2000).

[36] Berne, R. M. and Levy, M. N. Cardiovascular Physiology 6th ed. (C.V. Mosby, St. Louis, 1996).

[37] C.-K. Peng, S. V. Buldyrev, S. Havlin, M. Simons, H. E. Stanley, and A. L. Goldberger, "On the Mosaic Organization of DNA Sequences," Phys. Rev. E 49, 1691 (1994).

[38] K. Hu, P.Ch. Ivanov, C. Zhi, P. Carpena, and H.E. Stanley, "Effects of Trends on Detrended Fluctuation Analysis," Phys. Rev. E 64(1), (1 July, 2001) in press.

[39] S. V. Buldyrev, A. L. Goldberger, S. Havlin, R. N. Mantegna, M. E. Matsa, C.-K. Peng, M. Simons, and H. E. Stanley, "Long-Range Correlation Properties of Coding and Noncoding DNA Sequences: GenBank Analysis," Phys. Rev. E 51, 5084 (1995).

[40] S. V. Buldyrev, A. L. Goldberger, S. Havlin, C.-K. Peng, H. E. Stanley, and M. Simons, "Fractal Landscapes and Molecular Evolution: Modeling the Myosin Heavy Chain Gene Family," Biophys. J. 65, 2673 (1993).

[41] S. M. Ossadnik, S. V. Buldyrev, A. L. Goldberger, S. Havlin, R. N. Mantegna, C.-K. 
Peng, M. Simons, and H. E. Stanley, "Correlation Approach to Identify Coding Regions in DNA Sequences," Biophys. J. 67, 64 (1994).

[42] M. S. Taqqu, V. Teverovksy, and W. Willinger, "Estimators for Long-Range Dependence: an empirical study," Fractals 3, 185 (1996).

[43] H.V. Huikuri, K.M. Kessler, E. Terracall, A. Castellanos, M.K. Linnaluoto, R.J. Myerburg, "Reproducibility and circadian rhythm of heart rate variability in healthy subjects", Am. J. Cardiol. 65, 391 (1990).

[44] H. Moelgaard, K.E. Soerensen, and P. Bjerregaard, "Circadian Variation and Influence of Risk Factors on Heart Rate Variability in Healthy Subjects", Am. J. Cardiol. 68, 777 (1991).

[45] P.Ch. Ivanov M. G. Rosenblum, C.-K. Peng, S. Havlin, H.E. Stanley, and A.L. Goldberger, "Wavelets in Medicine and Physiology"' in Wavelets in Physics, ed. H. van der Berg (Cambridge University Press, Cambridge, 1998).

[46] Heart Failure Database (Beth Israel Deaconess Medical Center, Boston, MA). The database now includes 18 healthy subjects (13 female and 5 male, with ages between 20 and 50, average 34.3 years), and 12 congestive heart failure subjects ( 3 female and 9 male, with ages between 22 and 71, average 60.8 year) in sinus rhythm. Data is freely available at the following URL: http://www.physionet.org.

[47] P.Ch. Ivanov, A. Bunde, L.A.N. Amaral, S. Havlin, J. Fritsch-Yelle, R.M. Baevsky, H.E. Stanley and A.L. Goldberger, "Sleep-Wake Differences in Scaling Behavior of the Human Heartbeat: Analysis of Terrestrial and Long-Term Space Flight Data," Europhysics Letters 48, 594 (1999).

[48] A.L. Goldberger, M.W. Bungo, R.M. Baevsky, B.S. Bennett, D.R. Rigney, J.E. Mietus, G.A. Nikulina, and J.B. Charles, "Heart rate dynamics during long-term space flight: Report on MIR cosmonauts", Am. Heart J. 128, 202 (1994). 
[49] A. Bunde, S. Havlin, J.W. Kantelhardt, T. Penzel, J.H. Peter, K. Voigt, "Correlated and Uncorrelated Regions in Heart-Rate Fluctuations During Sleep", Phys. Rev. Lett. 85, $3736(2000)$.

[50] F. Mallamace and H. E. Stanley, eds., Physics of Complex Systems: Proc. Enrico Fermi School on Physics, Course CXXXIV (IOS Press, Amsterdam, 1997).

[51] P. Meakin, Fractals, Scaling and Growth Far from Equilibrium (Cambridge University Press, Cambridge, 1997).

[52] H.E. Stanley, "Power Laws and Universality", Nature 378, 554 (1995).

[53] A. Bunde and S. Havlin, Fractals in science (Springer-Verlag, Berlin, 1994).

[54] A. Bunde and S. Havlin, eds., Fractals and Disordered Systems, 2nd Edition (SpringerVerlag, Berlin, 1996).

[55] T. Vicsek, Fractal Growth Phenomena, 2nd ed. (World Scientific, Singapore, 1993).

[56] A.-L. Barabási and H. E. Stanley, Fractal Concepts in Surface Growth (Cambridge University Press, Cambridge, 1995).

[57] H. Takayasu, Fractals in the Physical Sciences (Manchester University Press, Manchester UK, 1997).

[58] H.E. Hurst, "Long-term Storage Capacity of Reservoirs", Trans. Am. Soc. Civ. Eng. 116, 770 (1951).

[59] T.G. Dewey, Fractals in Molecular Biophysics (Oxford University Press, Oxford, 1997).

[60] Z. R. Struzik, "Determining Local Singularity Strengths and their Spectra with the Wavelet Transform", Fractals, 8(2) (2000).

[61] Z. R. Struzik, "Revealing Local Variability Properties of Human Heartbeat Intervals with the Local Effective Hölder Exponent", Fractals, 9(1) (2001). 
[62] T. Vicsek and A.L. Barabási, "Multi-affine Model for the Velocity Distribution in Fully Turbulent Flows", J. Phys. A: Math. Gen., 24, L845 (1991).

[63] A.-L. Barabasi and H.E. Stanley, Fractal Concepts in Surface Growth (Cambridge University Press, Cambridge, 1995), Chapter 24.

[64] I. Daubechies, Ten Lectures on Wavelets (S.I.A.M., Philadelphia, 1992).

[65] J.F. Muzy, E. Bacry and A. Arneodo, "The Multifractal Formalism Revisited with Wavelets", Int. J. Bifurc. Chaos. 4, 245 (1994).

[66] L.A.N. Amaral, P.Ch. Ivanov, N. Aoyagi, I. Hidaka, S. Tomono, A.L. Goldberger, H.E. Stanley, and Y. Yamamoto, "Behavioral-Independent Features of Complex Heartbeat Dynamics", Phys. Rev. Lett. 86, (25 June, 2001) in press.

[67] L. A. N. Amaral, A. L. Goldberger, P. Ch. Ivanov, and H. E. Stanley, "Scale-Independent Measures and Pathologic Cardiac Dynamics", Phys. Rev. Lett. 81, 2388 (1998).

[68] C. Meneveau and K. R. Sreenivasan, "Simple Multifractal Cascade Model for Fully Developed Turbulence", Phys. Rev. Lett. 59, 1424 (1987).

[69] H.E. Stanley and P. Meakin, "Multifractal Phenomena in Physics and Chemistry", Nature 335, 405 (1988).

[70] U. Frisch, Turbulence (Cambridge University Press, Cambridge UK, 1995).

[71] L. Glass and C.P. Malta, "Chaos in Multiloop Negative Feedback-Systems", J. Theor. Biol. 145(2), 217 (1990).

[72] H. Seidel and H. Herzel, "Bifurcations in a Nonlinear Model of the Baroreceptor-Cardiac Reflex," Physica D, 115(1-2), 145 (1998).

[73] D.C. Lin and R.L. Hughson, "Modeling Heart Rate Variability in Healthy Humans: A Turbulence Analogy", Rev. Lett. 86, 1650 (2001) 


\section{FIGURES}

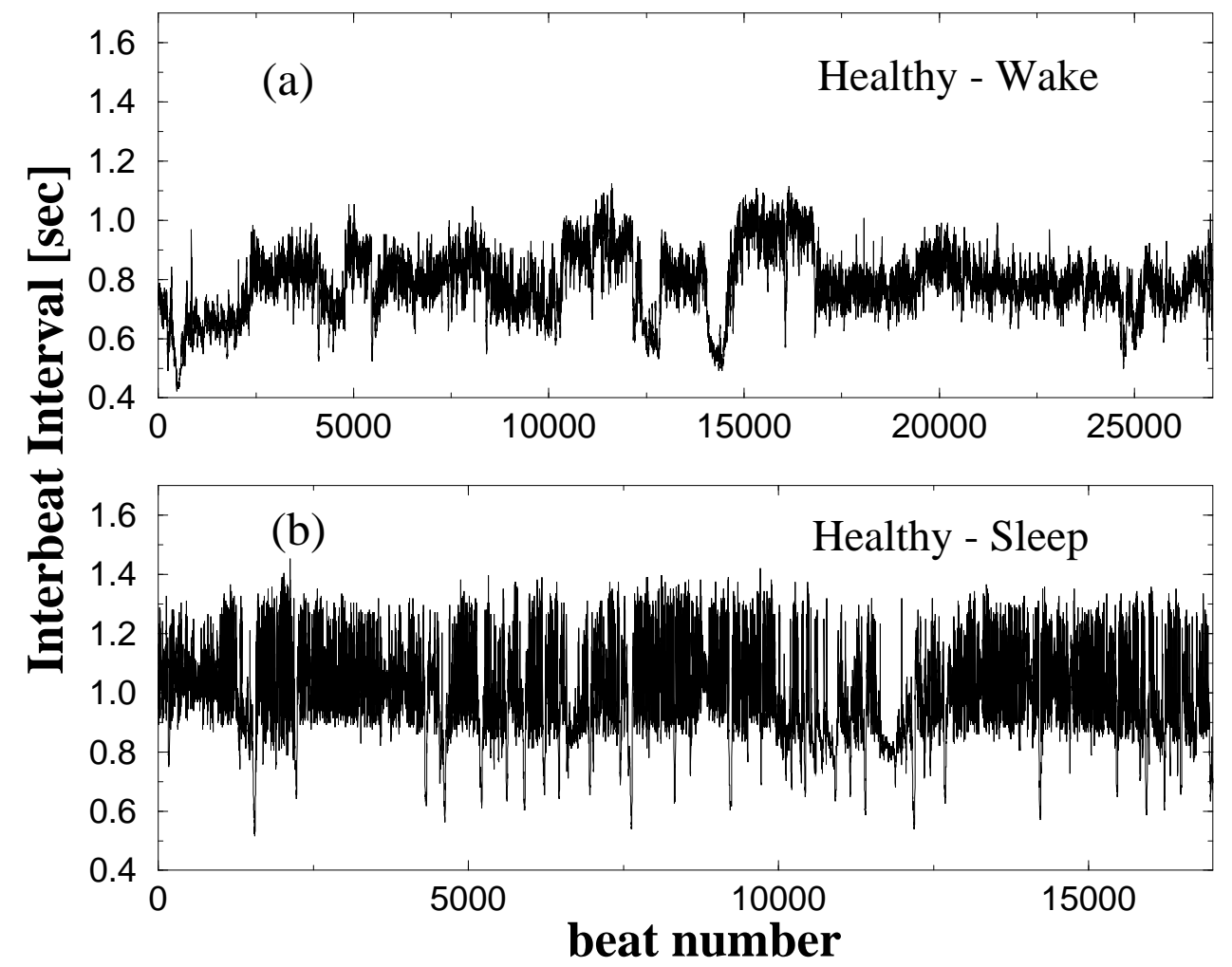

FIG. 1. Consecutive heartbeat intervals are plotted vs beat number for 6 hours recorded from the same healthy subject during: (a) wake period: $12 \mathrm{pm}$ to $6 \mathrm{pm}$ and (b) sleep period: $12 \mathrm{am}$ to 6am. (Note that there are fewer interbeat intervals during sleep due to the larger average of the interbeat intervals, i.e. slower heart rate.) 

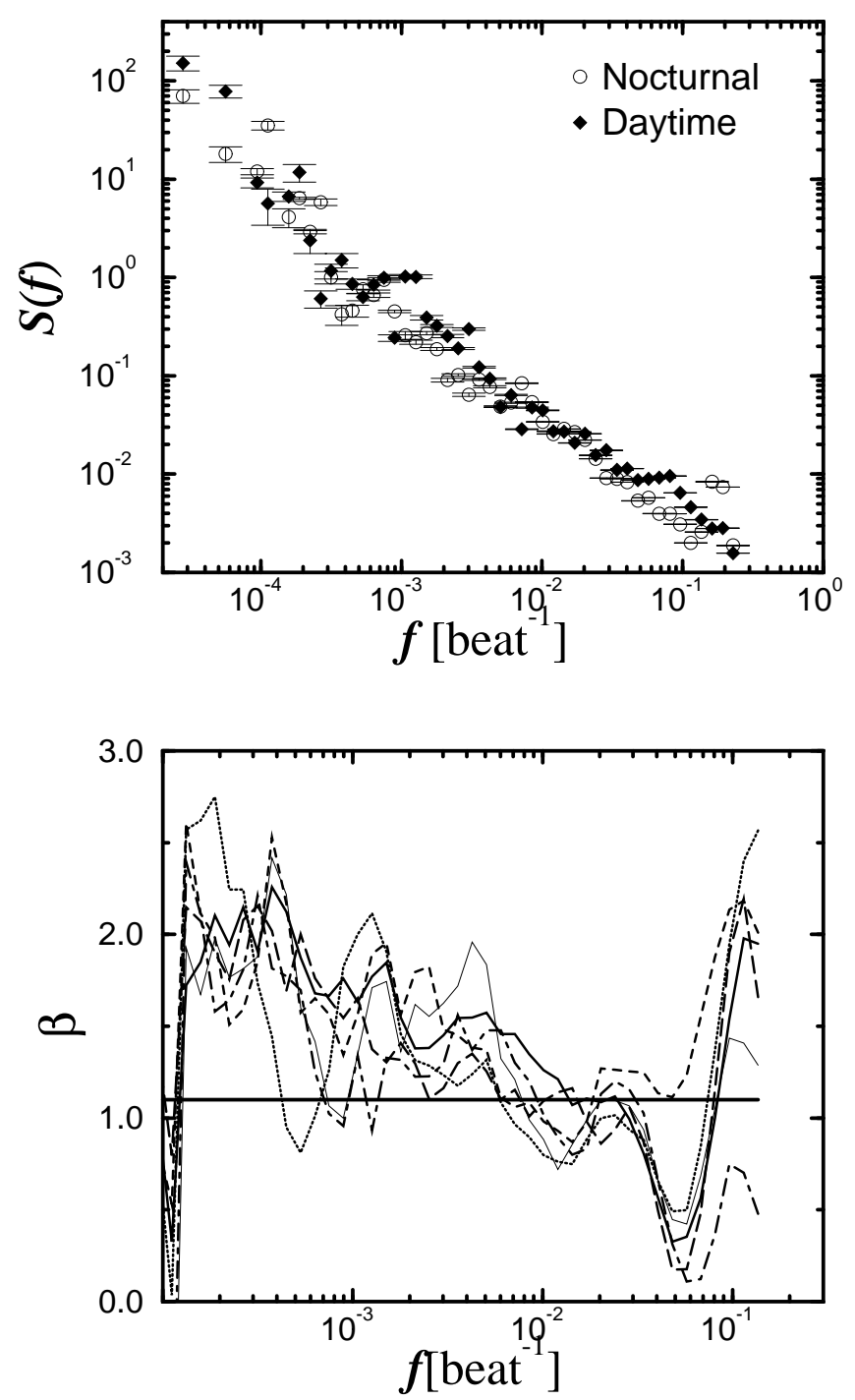

FIG. 2. (top) Power spectrum from 6 hour records of interbeat intervals for a healthy subject during day and night. (bottom) We plot the local exponent $\beta$ calculated from the power spectrum for 6 healthy subjects. The local value of $\beta$ shows a persistent drift, so no true scaling exists. This is not surprising, having in mind the non-stationarity of the signals. The horizontal line shows the value of the exponent obtained from a least square fit to the data. 

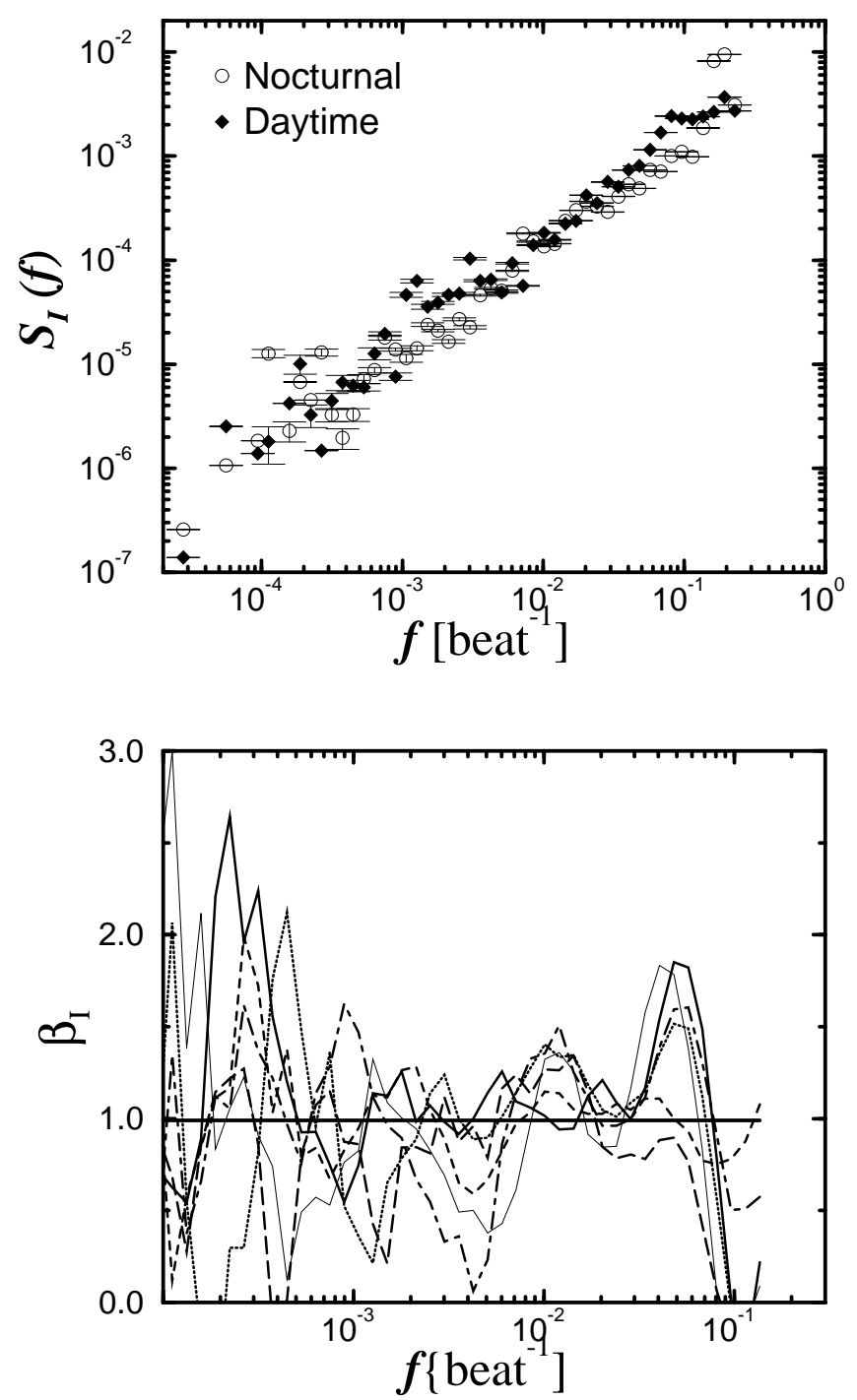

FIG. 3. (top) Power spectrum of the interbeat interval increments from $6 \mathrm{~h}$ record for the same healthy subject as in Fig. 2. Error bars are calculated as the standard deviation of the power spectrum values for frequencies within the binning interval. (bottom)The local exponent $\beta_{I}$ for the power spectrum of the increments for the same 6 healthy subjects as in Fig. 2. Note that the exponent $\beta_{I}$ fluctuates around an average value close to one, so true scaling does exist. The horizontal line shows the value of $\beta_{I}$ obtained from a least square fit. Note however, that the difference between wake and sleep dynamics cannot be observed from the power spectra. 

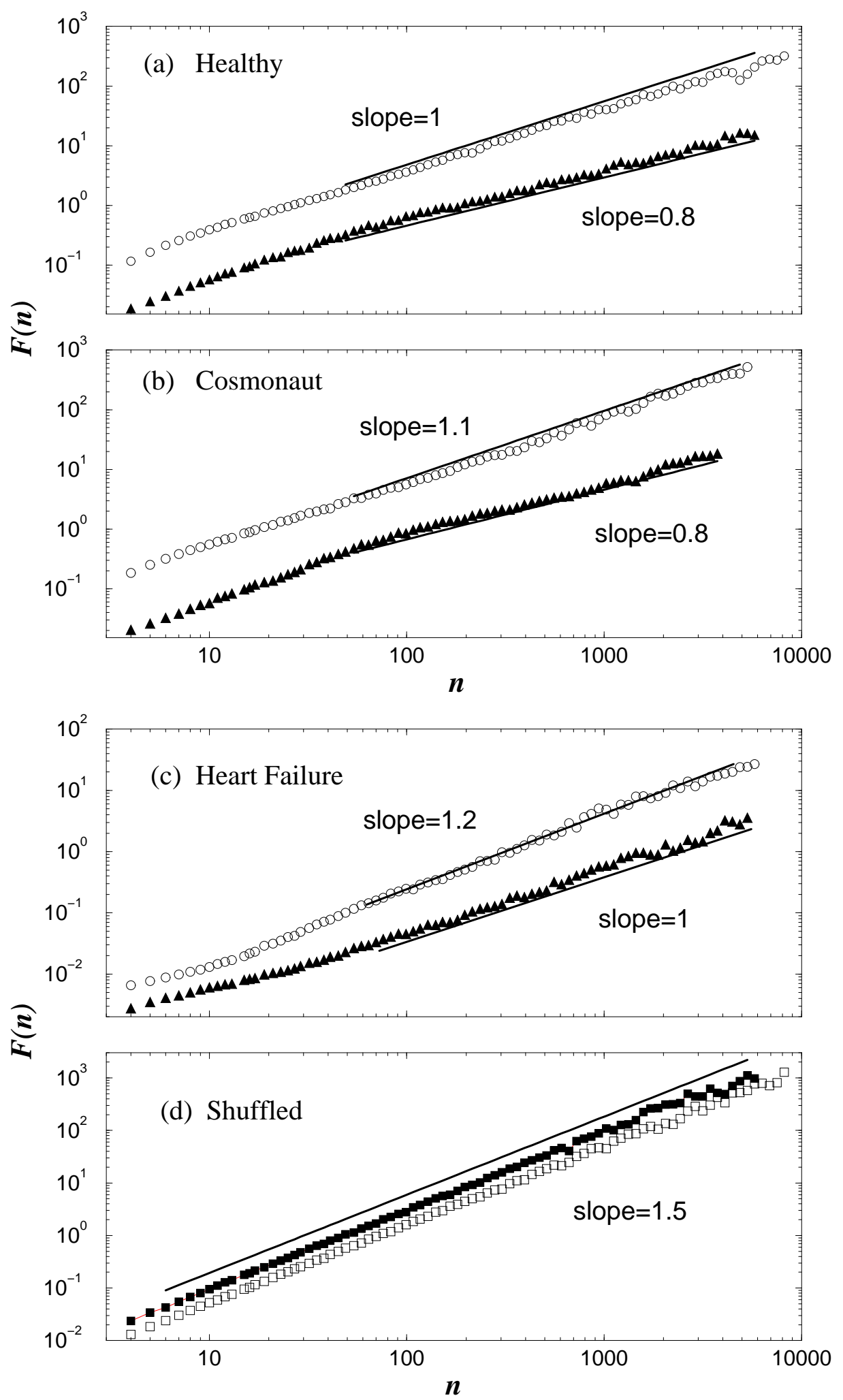
FIG. 4. Plots of $\log F(n)$ vs. $\log n$ for 6 h wake (open circles) and sleep records (filled triangles) of (a) one typical healthy subject; (b) one cosmonaut (during orbital flight); and (c) one patient with congestive heart failure. Note the systematic lower exponent for the sleep phase (filled triangles), indicating stronger anticorrelations. (d) As a control, we reshuffle and integrate the interbeat increments from the wake (open squares) and sleep data (solid squares) of the healthy subject presented in (a). We find a Brownian noise scaling over all time scales for both wake and sleep phases with an exponent $\alpha=1.5$, as one expects for random walk-like fluctuations. 


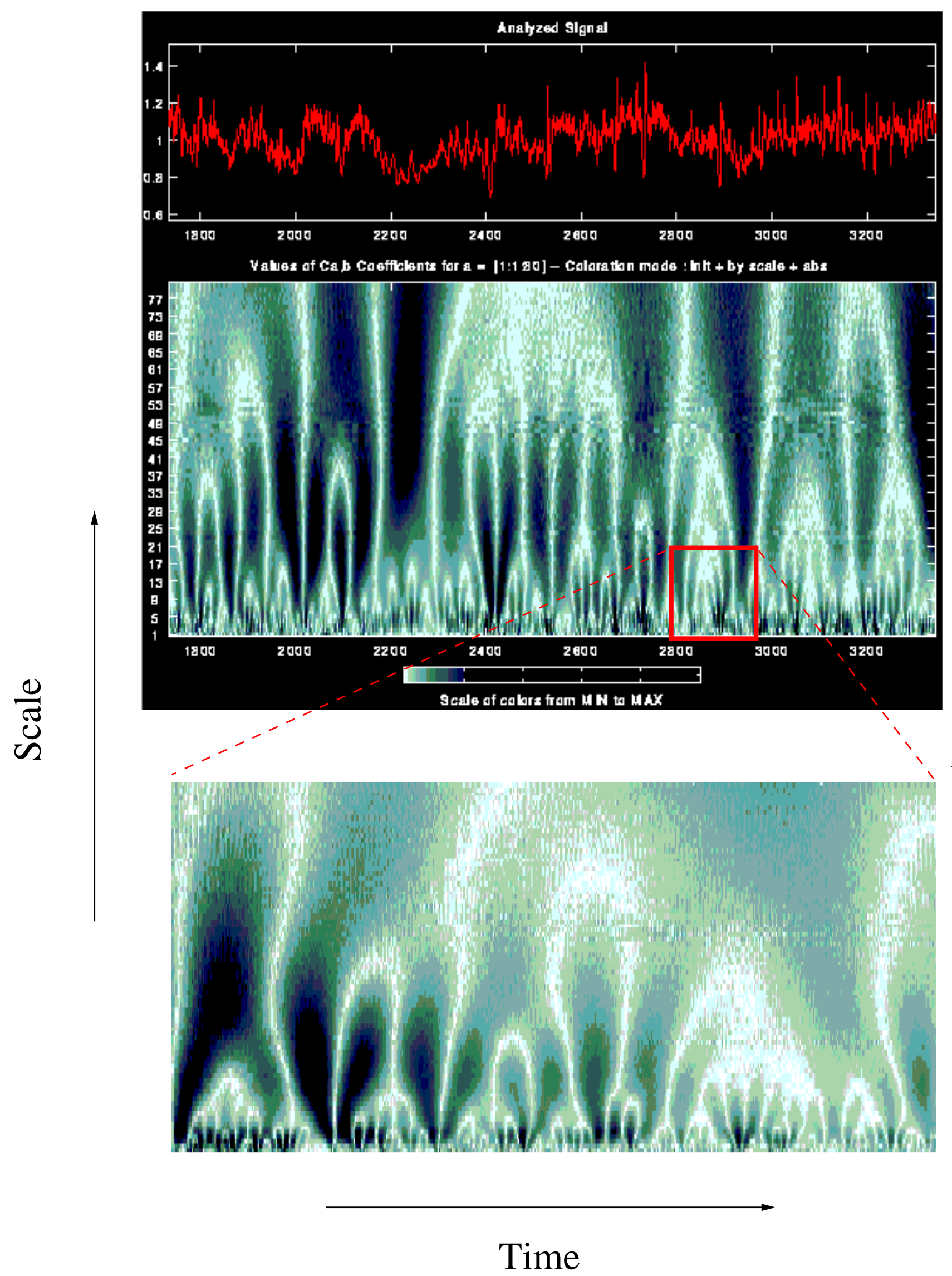


FIG. 5. Color coded wavelet analysis of a heartbeat interval signal. The $x$-axis represents time $(\approx 1700$ beats $)$ and the $y$-axis indicates the scale of the wavelet used $(a=1,2, \ldots, 80 ;$ i.e. $\approx$ from 5 seconds to 5 minutes) with large scales at the top. This wavelet decomposition reveals a self-similar fractal structure in the healthy cardiac dynamics - a magnification of the central portion of the top panel with 200 beats on the $x$-axis and wavelet scale $a=1,2, \ldots, 20$ on the $y$-axis shows similar branching patterns (lower panel). 

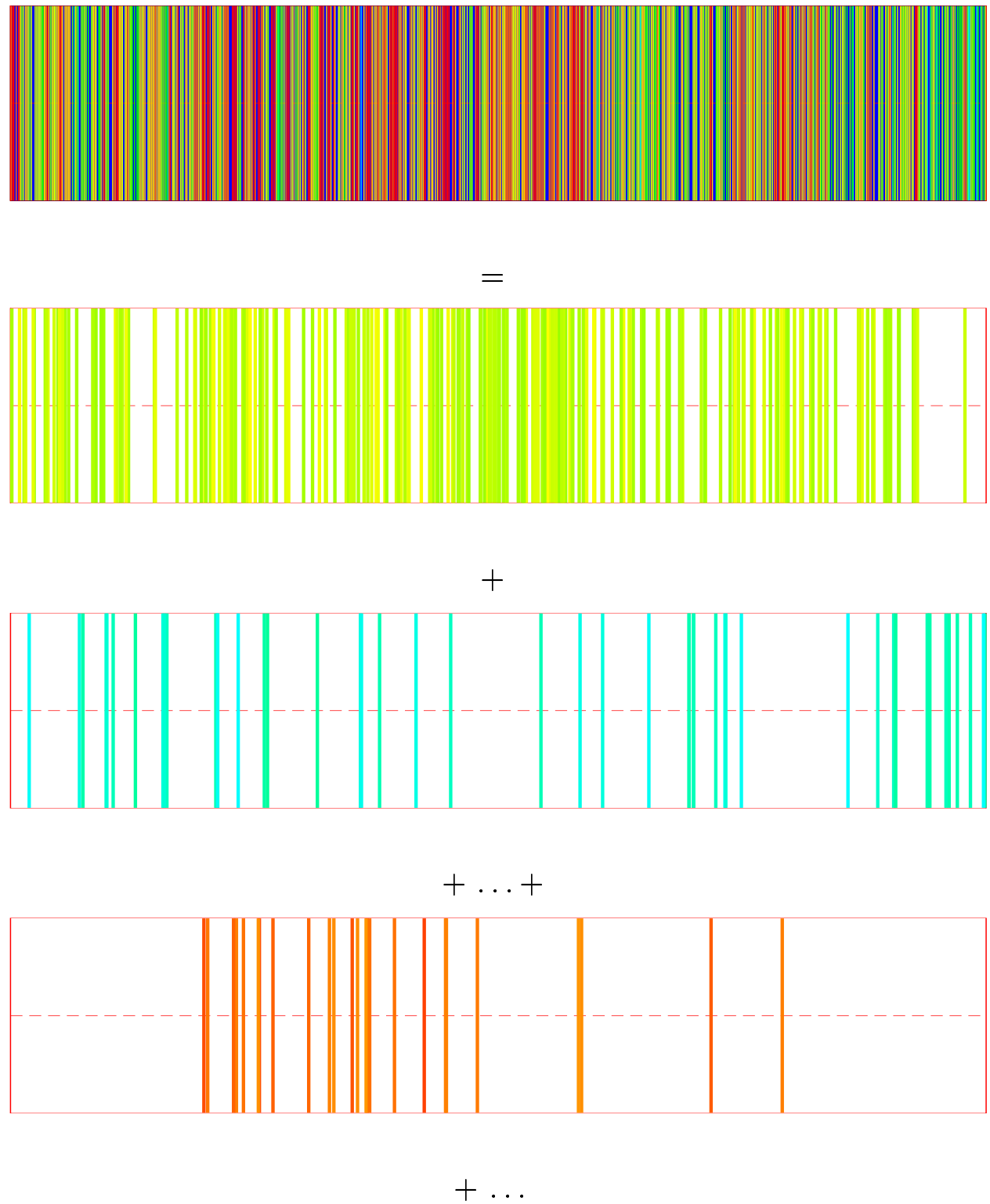

FIG. 6. Local Hurst exponents $h$ for a multifractal signal (top panel) and the decomposition of this signal into subsets (subsequent panels) with each local Hurst exponent indicated by the color and each fractal dimension indicated by the density of vertical bars. The $\mathrm{x}$-axis represents time and the vertical bars (y-axis) indicate local Hurst exponents. 


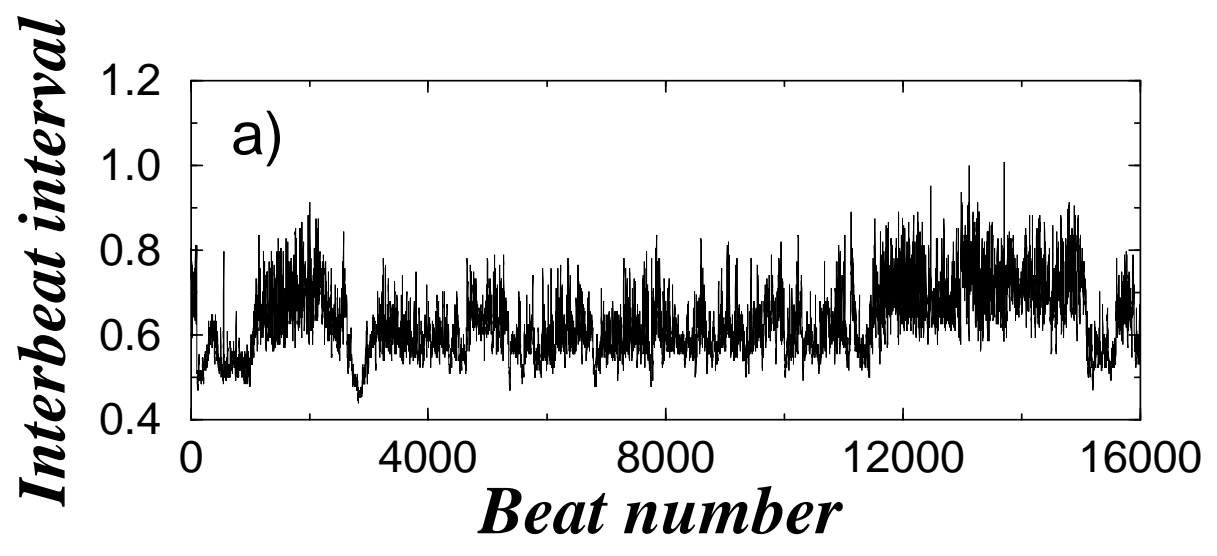

b)
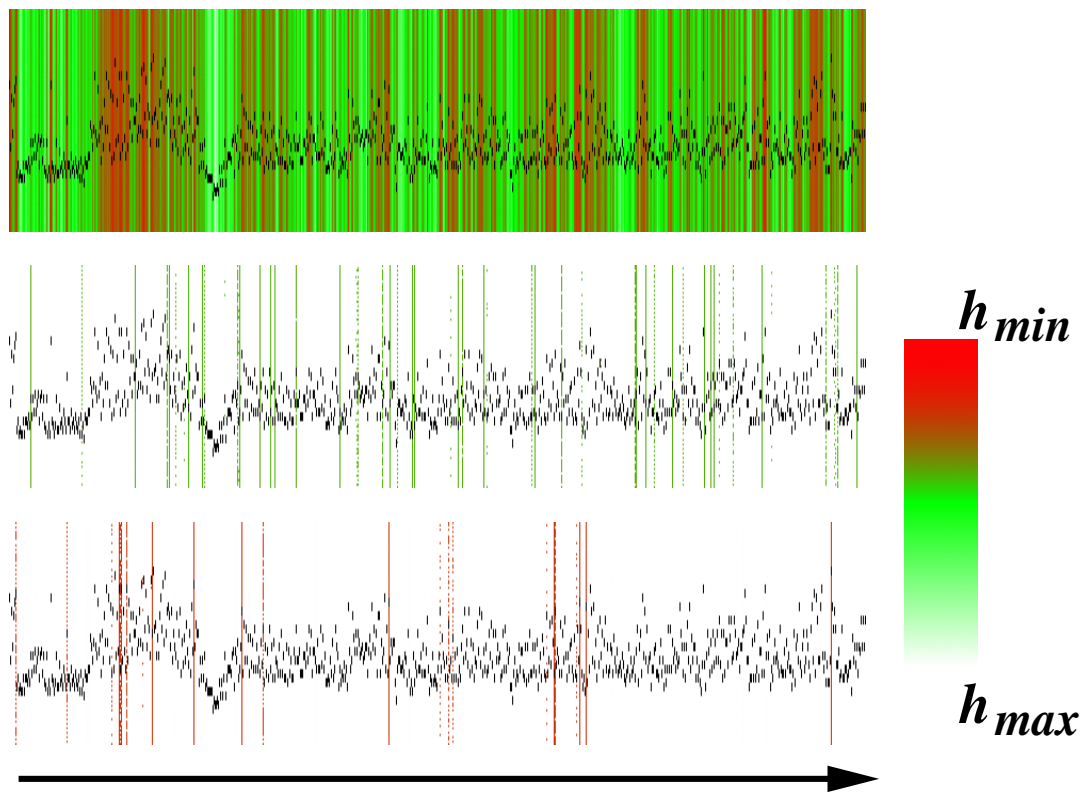

\section{Beat number}

c)

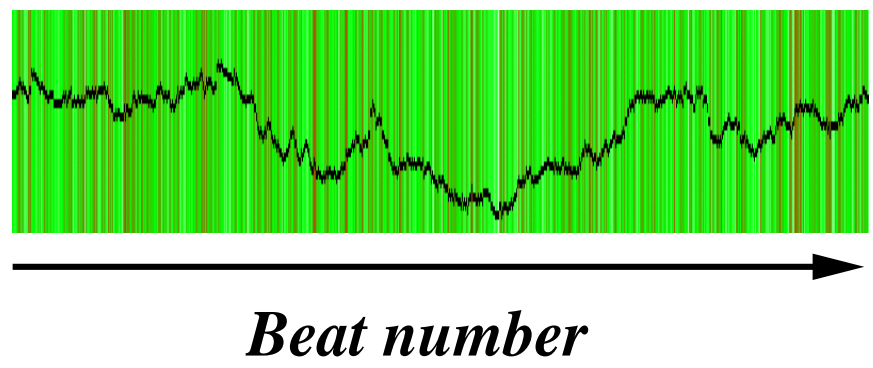


FIG. 7. (a) Consecutive heartbeat intervals measured in seconds are plotted vs beat number from approximately 3 hours record of a representative healthy subject. The time series exhibits very irregular and nonstationary behavior. (b) The top panel displays in color the local Hurst exponents calculated for the same 3 hours record shown in (a). The other two panels represent two subsets of the heartbeat interval time series in (a) each with a local Hurst exponent (indicated by the color) and with a different fractal dimension (indicated by the density of the vertical bars). (c) The panel displays in color the local Hurst exponents calculated for a monofractal signal fractional Brownian motion with $H=0.6$. The homogeneity of the signal is represented by the nearly monochromatic appearance of the signal which indicates that the local Hurst exponent $h$ is the same throughout the signal and identical to the global Hurst exponent $H$. 

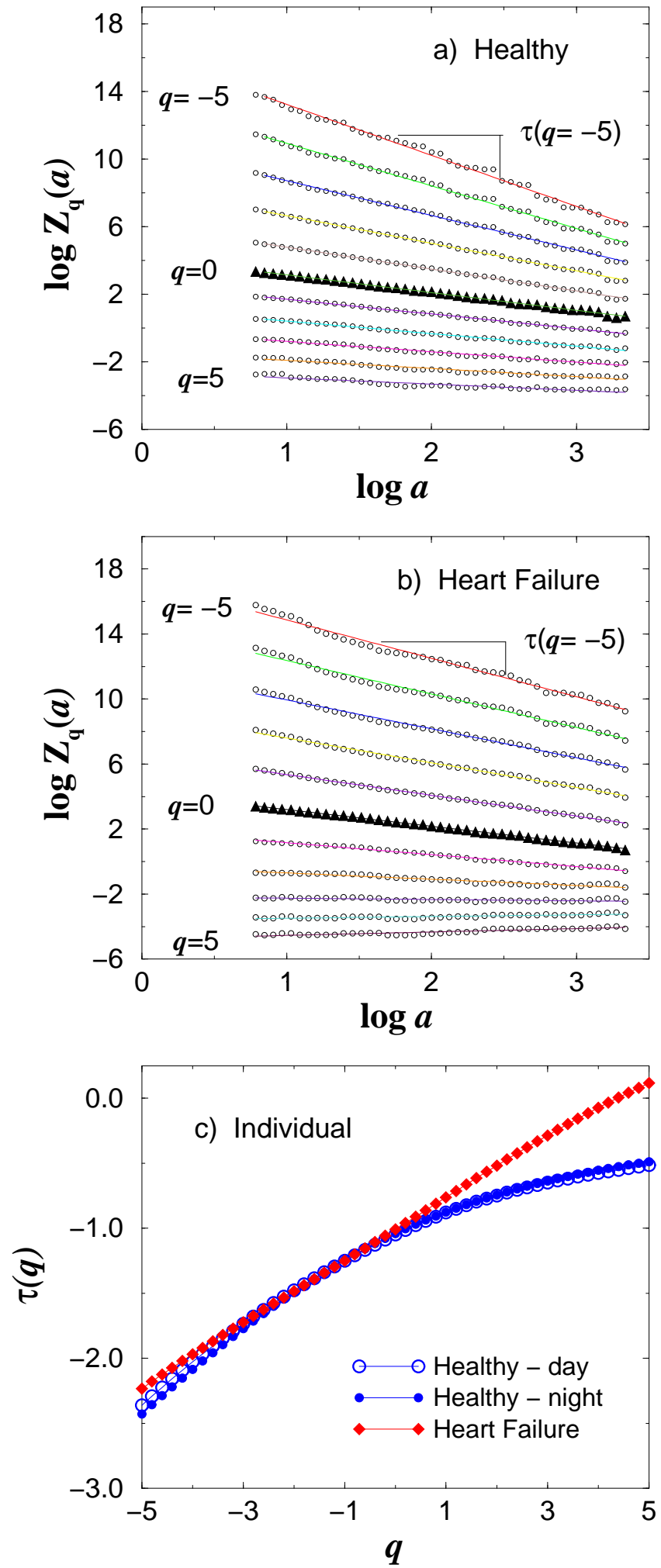
FIG. 8. Scaling of the partition function $Z_{q}(a)$ with scale $a$ obtained from daytime records consisting of $\approx 25,000$ beats for (a) a healthy subject and (b) a subject with congestive heart failure. (c) Multifractal spectrum $\tau(q)$ for the individual records in (a) and (b). 

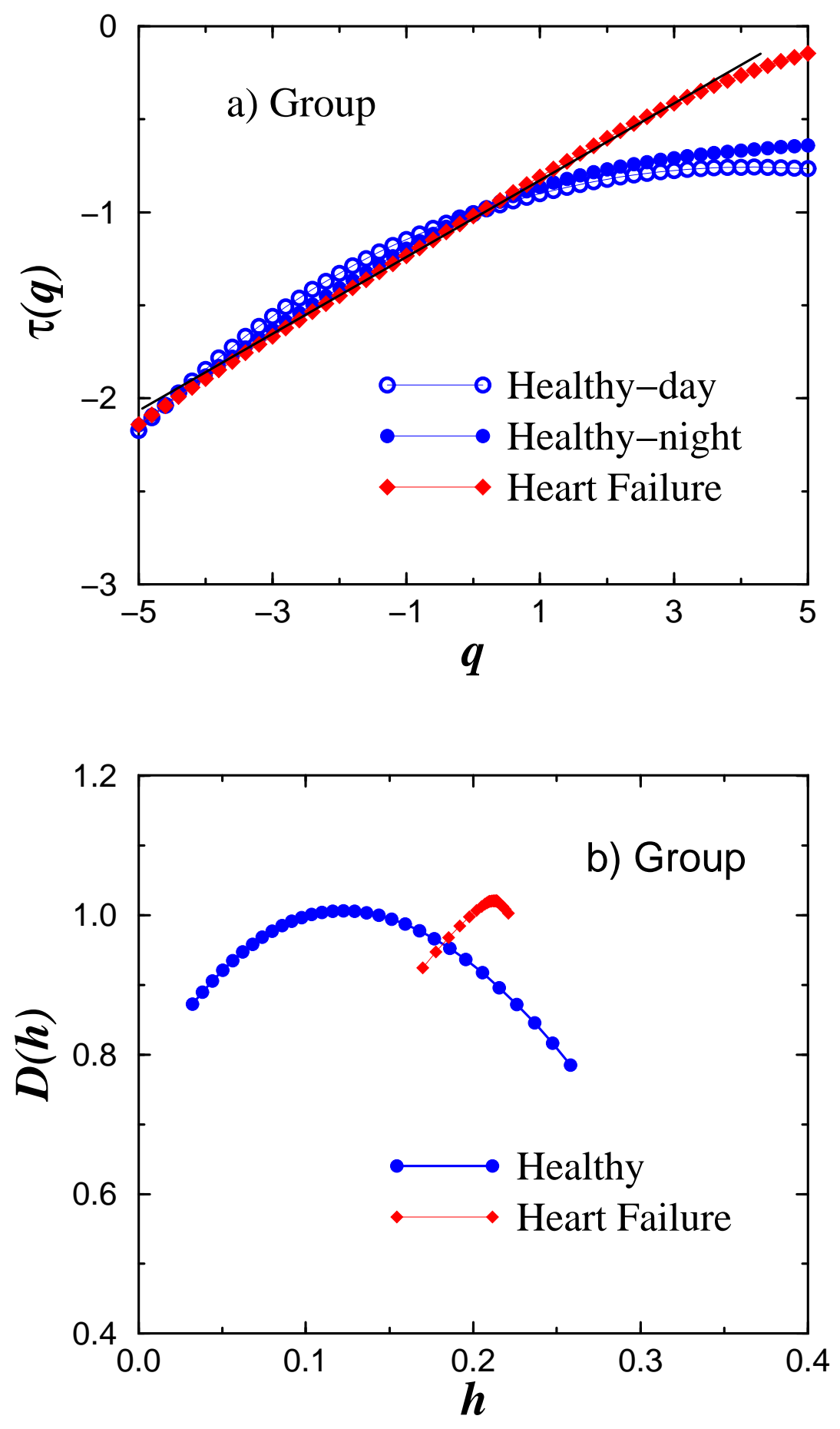
FIG. 9. (a) Multifractal spectrum $\tau(q)$ of the group averages for daytime and nighttime records for 18 healthy subjects and for 12 patients with congestive heart failure. The results show multifractal behavior for the healthy group and distinct change in this behavior for the heart failure group. (b) Fractal dimensions $D(h)$ obtained through a Legendre transform from the group averaged $\tau(q)$ spectra of (a). The shape of $D(h)$ for the individual records and for the group average is broad $(\Delta h \approx 0.25)$, indicating multifractal behavior. On the other hand, $D(h)$ for the heart failure group is very narrow $(\Delta h \approx 0.05)$, indicating loss of multifractality. The different form of $D(h)$ for the heart failure group may reflect perturbation of the cardiac neuroautonomic control mechanisms associated with this pathology. Note, that for $q=2$ the heartbeat fluctuations of healthy subjects are characterized by $h \approx 0.1$, which corresponds to $\alpha \approx 1.1$ for the interbeat interval series obtained from DFA analysis (Section III). 

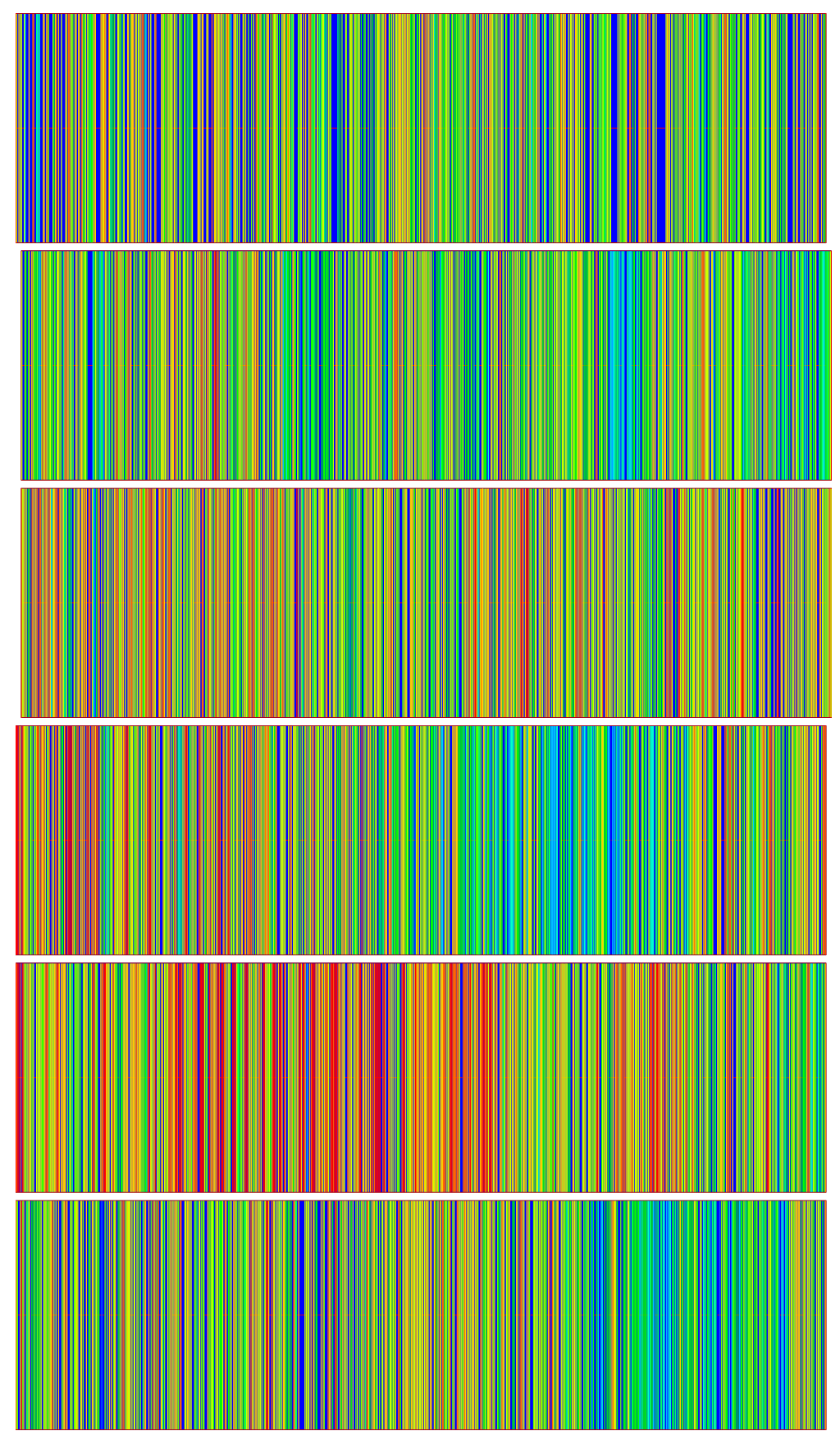

FIG. 10. Panels obtained from healthy individuals illustrating how the local Hurst exponent $h$ (vertical color bars) changes with time (x-axis). Each panel represents a $6 \mathrm{~h}$ record. A broad range of colors indicates broad multifractal spectrum. 

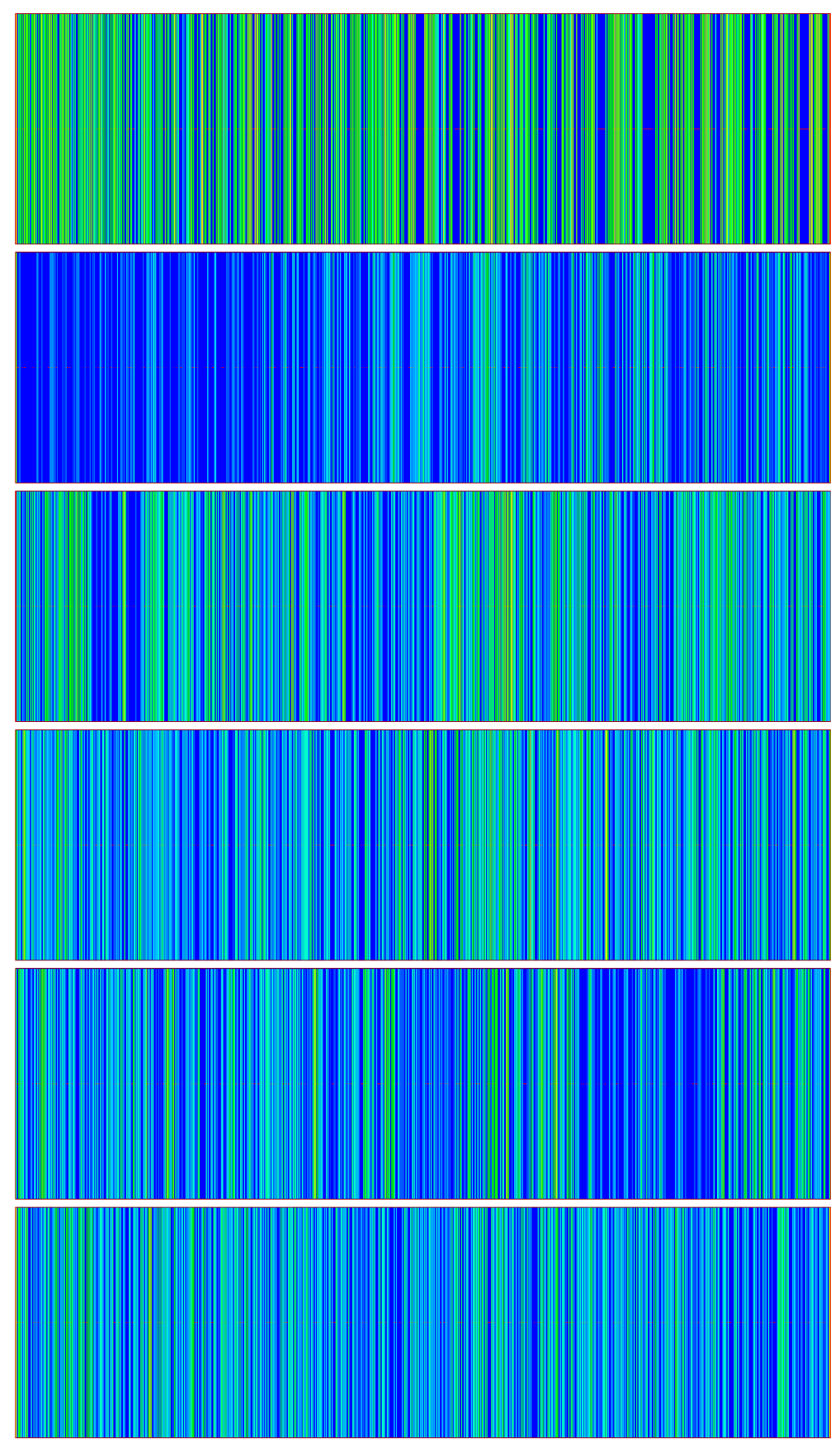

FIG. 11. Panels obtained from subjects with congestive heart failure illustrating how the local Hurst exponent $h$ (vertical color bars) changes with time (x-axis). Each panel represents a $6 \mathrm{~h}$ record. An almost monochromatic appearance indicates narrow multifractal spectrum, i.e. loss of multifractality. 

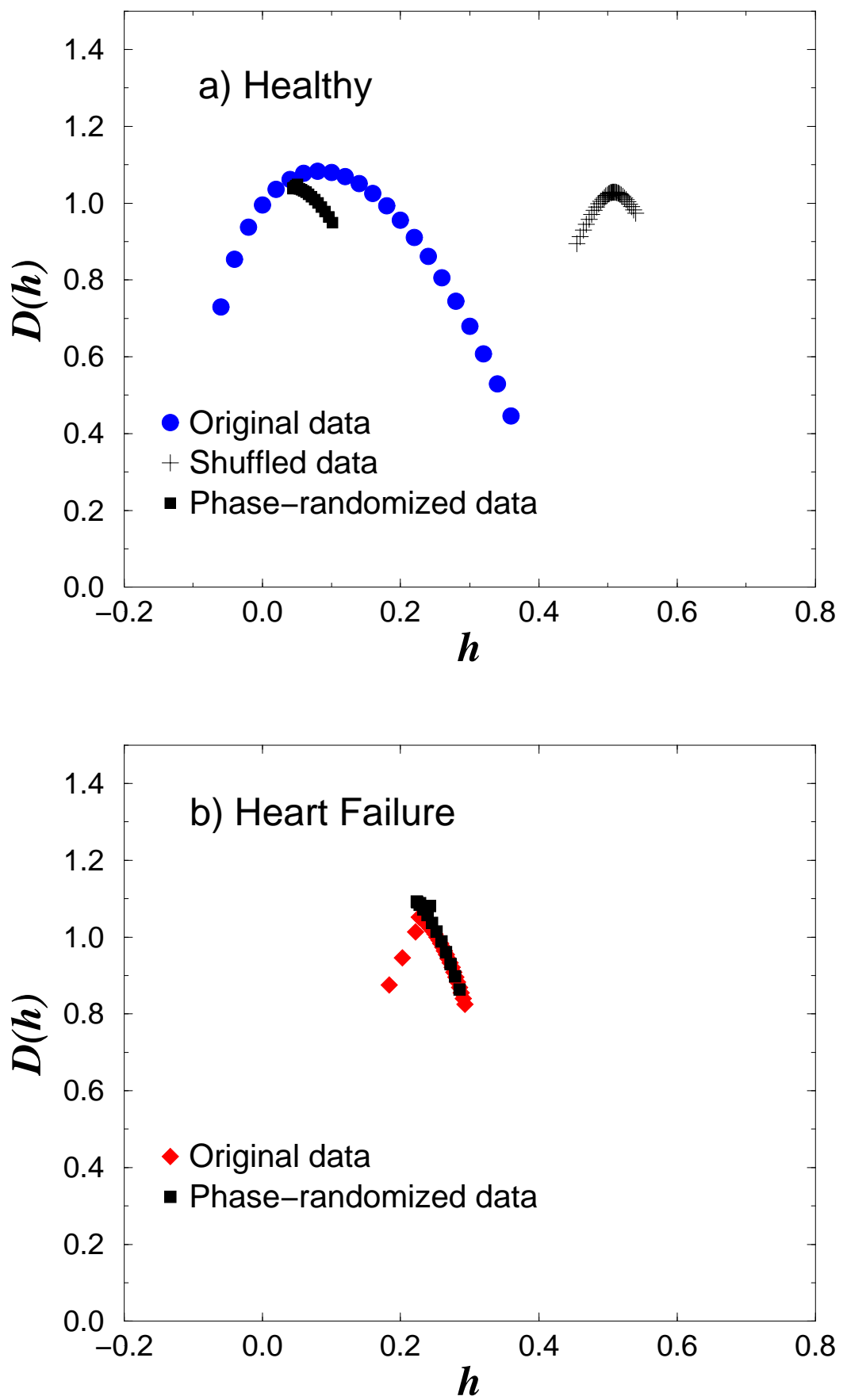
FIG. 12. (a) The fractal dimensions $D(h)$ for a $6 \mathrm{~h}$ daytime record of a healthy subject. After reshuffling and integrating the increments in this interbeat interval time series, so that all correlations are lost but the distribution is preserved, we obtain monofractal behavior - a very narrow point-like spectrum centered at $h \equiv H=1 / 2$. Such behavior corresponds to a simple random walk. A different test, in which the $1 / f$-scaling of the heart beat signal is preserved but the Fourier phases are randomized (i.e., nonlinearities are eliminated) leads again to a monofractal spectrum centered at $h \approx 0.07$, since the linear correlations were preserved. These tests indicate that the observed multifractality is related to nonlinear features of the healthy heart beat dynamics rather than to the ordering or the distribution of the interbeat intervals in the time series. (b) The fractal dimensions $D(h)$ for a $6 \mathrm{~h}$ daytime record of a heart failure subject. The narrow multifractal spectrum indicates loss of multifractal complexity and reduction of nonlinearities with pathology. 